ARTICLES nature medicine

\title{
Reversion of CTL escape-variant immunodeficiency viruses in vivo
}

\author{
Thomas C Friedrich ${ }^{1}$, Elizabeth J Dodds ${ }^{2}$, Levi J Yant ${ }^{2}$, Lara Vojnov ${ }^{2}$, Richard Rudersdorf ${ }^{1}$, Candice Cullen ${ }^{1}$, \\ David T Evans ${ }^{3}$, Ronald C Desrosiers ${ }^{3}$, Bianca R Mothé ${ }^{4}$, John Sidney ${ }^{5}$, Alessandro Sette ${ }^{5}$, Kevin Kunstman ${ }^{6}$, \\ Steven Wolinsky ${ }^{6}$, Michael Piatak ${ }^{7}$, Jeffrey Lifson ${ }^{7}$, Austin L Hughes ${ }^{8}$, Nancy Wilson ${ }^{1}$, David H O'Connor ${ }^{2}$ \& \\ David I Watkins ${ }^{1,2}$
}

Engendering cytotoxic T-lymphocyte (CTL) responses is likely to be an important goal of HIV vaccines. However, CTLs select for viral variants that escape immune detection. Maintenance of such escape variants in human populations could pose an obstacle to HIV vaccine development. We first observed that escape mutations in a heterogeneous simian immunodeficiency virus (SIV) isolate were lost upon passage to new animals. We therefore infected macaques with a cloned SIV bearing escape mutations in three immunodominant CTL epitopes, and followed viral evolution after infection. Here we show that each mutant epitope sequence continued to evolve in vivo, often re-establishing the original, CTL-susceptible sequence. We conclude that escape from CTL responses may exact a cost to viral fitness. In the absence of selective pressure upon transmission to new hosts, these original escape mutations can be lost. This suggests that some HIV CTL epitopes will be maintained in human populations.

Early attempts to design HIV vaccines focused on antibody responses, but the variability of the envelope proteins encoded by circulating virus strains has been a major obstacle to the development of broadly protective antibodies ${ }^{1-4}$. Recent vaccine approaches have focused instead on eliciting CTL responses ${ }^{5-7}$. CTLs are believed to have a major role in modulating the course of HIV disease. The appearance of CTL responses is temporally associated with the resolution of primary viremia in HIV-infected humans ${ }^{8,9}$, and depletion of $\mathrm{CD}^{+}$cells results in substantial increases in viral load in SIV-infected macaques ${ }^{10-12}$.

It is now well established that CTLs select for viral escape variants that are resistant to immune recognition ${ }^{13-24}$, but the fate of these escape mutations after transmission to new hosts is unclear. Escape from CTL responses can be associated with a loss of immune control of viral replication, in naive and vaccinated hosts ${ }^{14,16,17,25}$. One group has shown that escape-variant viruses can be transmitted from mothers to infants who shared the restricting human leukocyte antigen (HLA) element HLA-B27 (ref. 26). However, the majority of sexually transmitted HIV infections occur between individuals who express different HLA class I alleles. No study thus far has shown stable transmission of CTL escape viruses between HLA-disparate individuals, although some indirect evidence of such transmission has been reported ${ }^{24,26}$. If CTL escape mutations can be preserved after transmission of HIV between HLA class I-disparate individuals, it is likely that HIV escape variants are currently being propagated in populations. Over time, epitopes targeted by CTL-based vaccines could be lost from circulating virus strains, rendering vaccines based on single or consensus strains ineffective.

To determine the fate of CTL escape mutations after transmission of HIV, we modeled viral transmission between HLA-similar and HLAdisparate individuals using rhesus macaques infected with CTL escape-variant SIV. We first observed that CTL escape mutations were lost from a heterogeneous SIV isolate upon passage to animals lacking the major histocompatibility complex (MHC) class I determinants to recognize the mutant epitopes. These observations suggested that selection might favor reversion of CTL escape mutations in MHCdisparate hosts. However, it was also possible that we merely detected the stochastic outgrowth of different quasispecies, with no significant selective pressure exerted by MHC class I molecules. To test our hypothesis more rigorously, we challenged macaques with a clonal virus that harbored escape mutations in frequently recognized CTL epitopes, reasoning that any observed evolution of mutant epitope sequences would result from de novo mutations rather than the outgrowth of particular quasispecies present in the inoculum. We also compared the fitness of the engineered virus to that of wild-type SIV in vitro, using competitive coculture assays. Our results suggest that there is a previously unappreciated cost to viral fitness associated with escape from CTL responses.

${ }^{1}$ Wisconsin National Primate Research Center, Madison, Wisconsin 53715, USA. ${ }^{2}$ Department of Pathology and Laboratory Medicine, University of Wisconsin Medical School, Madison, Wisconsin 53706, USA. ${ }^{3}$ New England Primate Research Center, Southborough, Massachusetts 01772, USA. ${ }^{4}$ California State University, San Marcos, San Marcos, California 92096, USA. ${ }^{5}$ La Jolla Institute of Allergy and Immunology, San Diego, California 92121, USA. ${ }^{6}$ Northwestern University Medical School, Chicago, Illinois 60611, USA. ${ }^{7}$ AIDS Vaccine Program, National Cancer Institute, Frederick, Maryland 21702, USA. ${ }^{8}$ Department of Biological Sciences, University of South Carolina, Columbia, South Carolina 29208, USA. Correspondence should be addressed to D.I.W. (watkins@primate.wisc.edu).

Published online 15 February 2004; doi:10.1038/nm998 


\section{ARTICLES}

a

\begin{tabular}{|c|c|c|c|}
\hline $\mathrm{SIV}_{\operatorname{mac} 239}$ & $\begin{array}{cc}160 & 170 \\
\text { IPDWQDYTSGPGIRYPKTFGWLWKLVP }\end{array}$ & & \\
\hline Inoculum & ……WF & $\begin{array}{l}(15 / 26) \\
(11 / 26)\end{array}$ & \\
\hline & 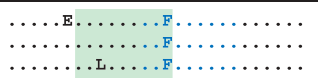 & $\begin{array}{l}(7 / 13) \\
(5 / 13) \\
(1 / 13)\end{array}$ & 3 weeks p.i. \\
\hline & 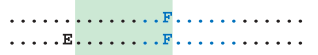 & $\begin{array}{l}(6 / 10) \\
(4 / 10)\end{array}$ & 29 weeks p.i. \\
\hline & 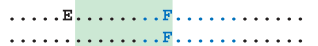 & $\begin{array}{l}(9 / 22) \\
(8 / 22)\end{array}$ & \\
\hline 88090 & $\ldots \ldots \ldots \ldots$ F & $(1 / 22)$ & \\
\hline$A^{*} 02-$ negative & $\ldots$.E. & $(1 / 22)$ & 49 weeks p.i. \\
\hline$B^{*} 17-$ negative & $\ldots \ldots \ldots \ldots \ldots+\cdots$ & $(1 / 22)$ & \\
\hline & 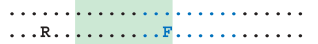 & $\begin{array}{l}(1 / 22) \\
(1 / 22)\end{array}$ & \\
\hline & … & $(27 / 49)$ & \\
\hline & 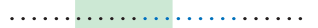 & $(11 / 49)$ & \\
\hline & $\ldots \ldots$ E. & $(5 / 49)$ & \\
\hline & $\ldots$ в. & $(4 / 49)$ & 61 weeks p.i. \\
\hline & м. $\ldots \ldots \ldots \ldots \ldots \ldots \ldots \ldots$ & $(1 / 49)$ & \\
\hline & $\ldots \ldots \ldots \ldots \ldots$ г. $\ldots \ldots \ldots$ & $(1 / 49)$ & \\
\hline & 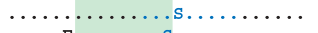 & $(1 / 49)$ & \\
\hline & 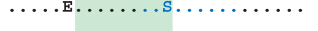 & $(1 / 49)$ & \\
\hline & $\ldots \ldots+$ & $(17 / 36)$ & \\
\hline & $\ldots \ldots$ в $\ldots \ldots \ldots \ldots \ldots \ldots$ & $(13 / 36)$ & \\
\hline & $\ldots \ldots \ldots \ldots \ldots+\ldots \ldots \ldots$ & $(4 / 36)$ & Necropsy (86 weeks p.l \\
\hline & $\ldots$ в. & $(1 / 36)$ & \\
\hline & $\ldots \ldots \ldots{ }^{*} \ldots \ldots$ E. $\ldots \ldots \ldots$ & $(1 / 36)$ & \\
\hline & … & $(6 / 12)$ & \\
\hline & 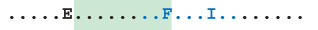 & $(2 / 12)$ & 11 weeks $\mathrm{pj}$ \\
\hline & 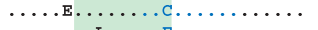 & $(3 / 12)$ & \\
\hline & 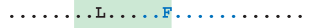 & $(1 / 12)$ & \\
\hline 95024 & 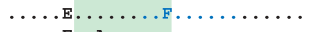 & $(6 / 10)$ & \\
\hline$A^{*} 02$-negative & 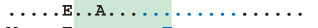 & $(1 / 10)$ & \\
\hline$B^{*} 17-n e g a t i v e$ & 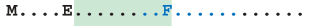 & $(1 / 10)$ & 35 weeks p.i. \\
\hline & $\ldots \ldots \ldots \ldots \ldots$ & $(1 / 10)$ & \\
\hline & 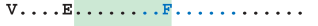 & $(1 / 10)$ & \\
\hline & $\ldots \ldots$ в. & $(9 / 12)$ & \\
\hline & $\ldots \ldots$ в. $\ldots$ v. $\ldots \ldots \ldots \ldots$ & $(2 / 12)$ & 84 weeks p.i. \\
\hline & 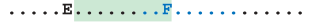 & $(1 / 12)$ & \\
\hline
\end{tabular}

Figure 1 Sequence variation in macaques challenged with $\operatorname{SIV}_{\mathrm{ppm}}$, a biological isolate with amino acid replacements in CTL epitopes restricted by common macaque $\mathrm{MHC}$ class I molecules Mamu-A*01 (Tat SL8), Mamu-A*02 (Nef YY9) and Mamu-B*17 (Nef IW9). (a) In animals lacking both Mamu-A*02 and Mamu- ${ }^{*} 17$, epitope sequences of both Nef YY9 (green shading) and Nef IW9 (blue letters) evolve toward wild type. p.i., post infection. (b) In animals expressing Mamu-B* 17 but not Mamu- $A^{*} 02$, only the region of Nef YY9 that does not overlap the $B * 17$ restricted Nef IW9 epitope evolves toward wild type. Variants in the $B * 17$-restricted epitope continue to evolve. (c) Oscillation occurs in tat codon 35 (C-terminal anchor of Tat SL8) in Mamu-A*01-negative animals infected with $\operatorname{SIV}_{\mathrm{ppm}}$. In contrast, there is further evolution away from $\mathrm{SIV}_{\text {mac239 }}$ epitope sequence in Mamu-A*01-positive animal (95024) infected with SIV $_{\mathrm{ppm}}$. Tat SL8 sequence is shaded in yellow.

\section{RESULTS}

\section{Escape mutations are lost from heterogeneous SIV in vivo}

To determine the fate of CTL escape mutations in transmitted virus variants, we examined the evolution of variant epitope sequences in five animals infected with a heterogeneous biological isolate, $\mathrm{SIV}_{\mathrm{ppm}}$ (ref. 19), that is derived from the well-characterized pathogenic clone, $\operatorname{SIV}_{\text {mac239 }}$ (ref. 27 and Supplementary Fig. 1 online). The SIV inoculum sequence encodes amino acid substitutions in CTL epitopes restricted by Mamu-A ${ }^{\star} 01$ ( Tat $_{28-35}$ SL8 (abbreviated Tat SL8); amino acid sequence STPESANL), Mamu-A ${ }^{\star} 02\left(\mathrm{Nef}_{159-167} \mathrm{YY} 9\right.$ (Nef YY9); YTSGPGIRY) and Mamu-B ${ }^{\star} 17\left(\mathrm{Nef}_{165-173}\right.$ IW9 (Nef IW9); IRYPKT- b

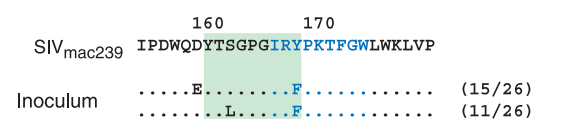

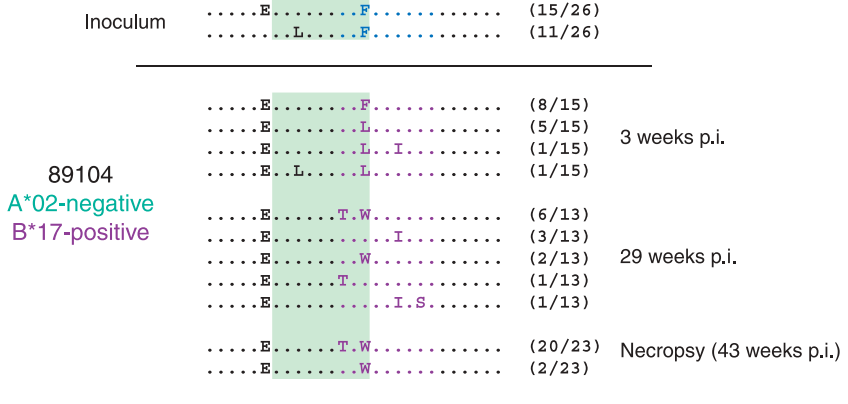

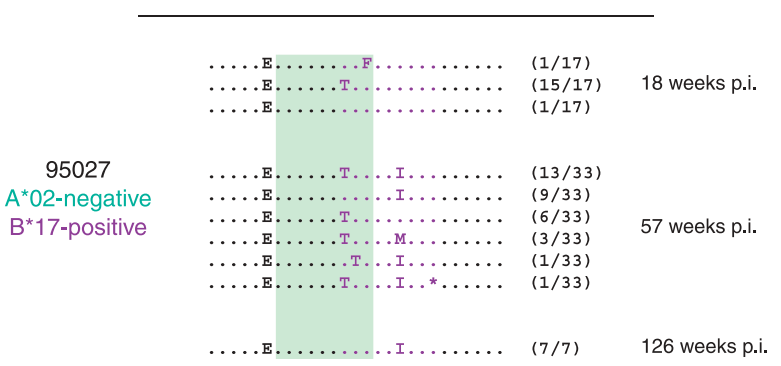

C
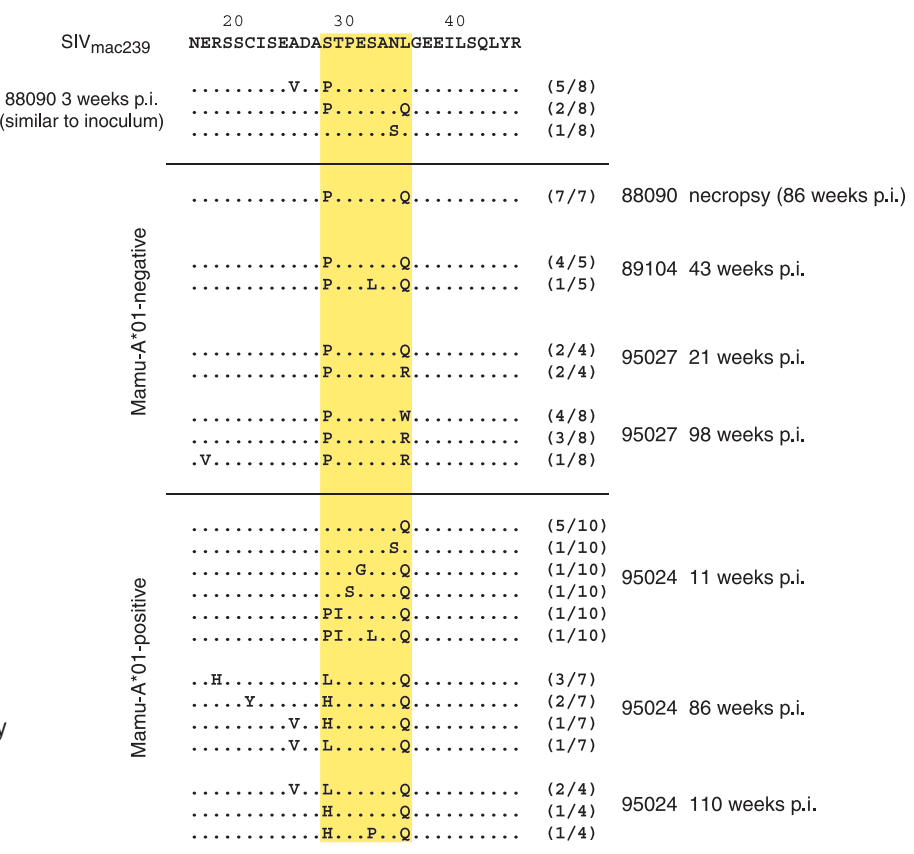

FGW). Two of the animals infected with this isolate were Mamu- $B^{*} 17$ positive, one was Mamu- $\mathrm{A}^{\star} 01$-positive, and none expressed Mamu$\mathrm{A}^{\star} 02$.

We first sequenced the overlapping Mamu- $\mathrm{A}^{*} 02$-restricted ( $\mathrm{Nef}$ YY9) and Mamu-B* 17 -restricted (Nef IW9) epitopes. In the two animals that expressed neither of these restricting elements, the epitope sequences of both Nef YY9 and Nef IW9 evolved toward the SIV mac239 $_{2}$ wild-type sequence (Fig. 1a). In contrast, in the two animals that expressed Mamu- $B^{\star} 17$ but not Mamu- $\mathrm{A}^{\star} 02$, further escape mutation was seen in the $B^{\star} 17$-restricted Nef IW9 epitope, whereas the $A^{\star} 02$ restricted Nef YY9 epitope again evolved toward wild type (Fig. 1b). We 
Table 1 Binding of wild-type and variant peptides to MHC class I

\begin{tabular}{lcccc}
\hline Epitope & Sequence & MHC class I & $I_{50}(\mathrm{nM})$ & \% reduction \\
\hline Gag CM9 & CTPYDINQM & Mamu-A*01 & 22 & - \\
Mutant & CAPPYINQM & Mamu-A*01 & 354 & 94 \\
Tat SL8 & STPESANL & Mamu-A*01 & 43 & - \\
Mutant & PTPESANQ & Mamu-A*01 & 25,535 & 99.9 \\
Nef IW9 & IRYPKTFFW & Mamu-B*17 & 7.6 & - \\
Mutant & TRYPKIFGW & Mamu-B*17 & 339 & 98 \\
\hline
\end{tabular}

Peptide binding to Mamu- $A^{*} 01$ or Mamu- ${ }^{*} 17$ molecules was assessed using epitope peptides or variants in competition assays with radiolabeled reference peptides, as described previously 37,38 . Reported are the concentration of epitope peptide at which bound radioactivity is reduced by $50 \%\left(\mathrm{IC}_{50}\right)$ and, for escape variants, percent reduction in binding with respect to wild-type peptide. Residues that differ between wild-type ans $3 x$ SIV are underlined.

next examined the sequences of Tat SL8. The sequence of tat in the inoculum was not available, but it is likely that the $\operatorname{SIV}_{\mathrm{ppm}}$ inoculum contained variation in the Mamu- $\mathrm{A}^{\star} 01$-restricted Tat SL8 epitope, as all sequences of this region isolated from a Mamu- $A^{\star} 01$-negative animal 3 weeks after infection differed from those of $\mathrm{SIV}_{\text {mac239 }}$ within the epitope. As $\mathrm{SIV}_{\mathrm{ppm}}$ infection progressed in Mamu- ${ }^{\star} 01$-negative animals, we detected oscillation in tat codon 35 , the C-terminal anchor of the epitope, but there was no reversion to wild-type sequences (Fig. 1c). We observed three different residues (glutamine, arginine and tryptophan) in tat codon 35 in Mamu- $A^{\star} 01$-negative macaques, none of which is predicted to allow epitope peptide binding to Mamu- $A^{\star} 01$. However, Tat SL8 sequences evolved even further away from SIV mac239 in Mamu- $A^{\star} 01$-positive animal 95024, with leucine and histidine replacing proline and the wild-type serine by late chronic infection. Together, these observations raise the possibility that escape mutants continue to evolve in MHC-disparate hosts and that, under certain circumstances, wild-type epitope sequences can be re-established.

\section{Construction of a cloned CTL escape virus}

To study the transmission and evolution of viral escape variants in vivo with greater rigor, we constructed a molecularly cloned mutant SIV that differed from $\mathrm{SIV}_{\operatorname{mac} 239}$ at only three defined epitopes recognized by high-frequency CTLs. The molecularly cloned virus enabled us to study the effects of CTL escape mutations in a viral genetic background free of other variations that might affect viral replication and/or pathogenicity in vivo. Moreover, because infections were initiated with a clonal sequence, any variation we detected would have to arise de novo within the infected animal, rather than reflecting the outgrowth of one or more members of a complex population of quasispecies present during initial infection.

We analyzed epitopes bound by two common Indian rhesus macaque MHC class I molecules, Mamu- ${ }^{\star} 01$ and Mamu-B ${ }^{\star} 17$. We have previously shown that macaques that express both Mamu- $\mathrm{A}^{\star} 01$ and $\mathrm{B}^{\star} 17$ are predisposed to unusual control of $\mathrm{SIV}_{\operatorname{mac} 239}$ replication, and produce three high-frequency (immunodominant) CTL responses during acute infection ${ }^{28}$. The epitopes recognized by these CTLs are derived from structural (Gag) and nonstructural (Tat and

Figure 2 Reduced fitness of 3x SIV in vitro. (a) 3x SIV lags behind wild-type (wt) virus in early replication kinetics. Results shown are from three separate trials with each virus inoculum. (b) Wild-type virus outgrows $3 x$ SIV when inoculated in equal amounts. Wild-type vRNA is expressed as percentage of total copies of vRNA in each sample. Results are representative of independent triplicate experiments. Three independent quantitative RTPCR/SSP reactions were performed for each experiment.
Nef) proteins, and accumulate escape mutations with varying kinetics. CTLs specific for Gag $_{181-189}$ CM9 (abbreviated Gag CM9; sequence CTPYDINQM) and Tat SL8 are immunodominant during acute infection of Mamu-A*01-positive macaques ${ }^{29}$. Gag CM9-specific CTLs select for escape variants intermittently and only during chronic infection $^{23,30}$, whereas Tat SL8-specific CTLs select for rapid viral escape within 4 weeks of infection ${ }^{20}$. CTLs recognizing Nef IW9 are immunodominant in Mamu-B ${ }^{\star} 17$-positive macaques and select for escape variants with intermediate kinetics, $\sim 16$ weeks after infection ${ }^{23}$.

The consensus escape mutation in the Gag CM9 epitope encoded a threonine-to-alanine substitution occurring at the secondary anchor position of the epitope (T182A), and reduced peptide binding to the Mamu- $A^{*} 01$ molecule by tenfold (Table 1 ). In late-stage virus sequences, the mutation encoding T182A was also associated with two nucleotide changes outside the epitope, each of which encoded a isoleucine-to-valine substitution (I161V and I206V). We have shown that these compensatory substitutions probably restore fitness to the T182A escape virus, and we therefore included them in our mutagenesis strategy $\mathrm{y}^{30,31}$.

The consensus escape sequence for Tat SL8 had substitutions in positions 1 and 8 (S28P and L35Q). Leu35 is the C-terminal anchor of the Tat SL8 peptide, and consequently this substitution essentially abrogates the peptide's binding to Mamu- $A^{\star} 01$ (Table 1). Consensus escape substitutions for Nef IW9 were mapped to positions 1 and 6 (I165T and T170I); this sequence was also observed in a previous study ${ }^{19}$. These substitutions reduced binding to Mamu-B ${ }^{\star} 17$ by $>90 \%$ (Table 1), but because position 6 is thought to interact with the T-cell receptor, this variant may also affect T-cell recognition.
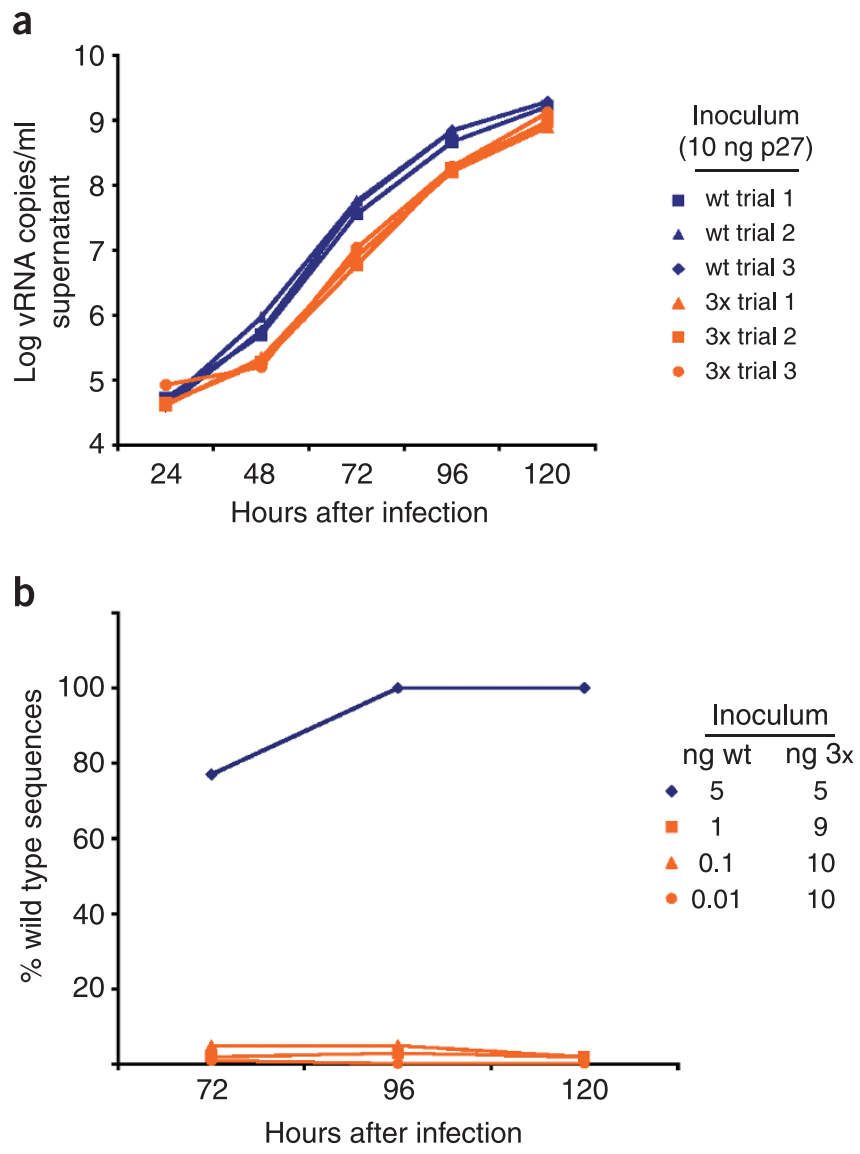


\section{ARTICLES}

a

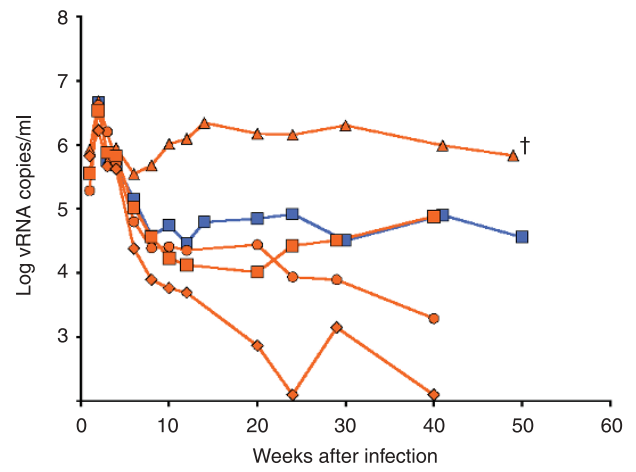

b

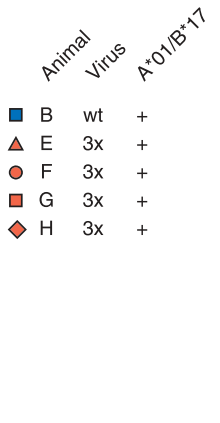

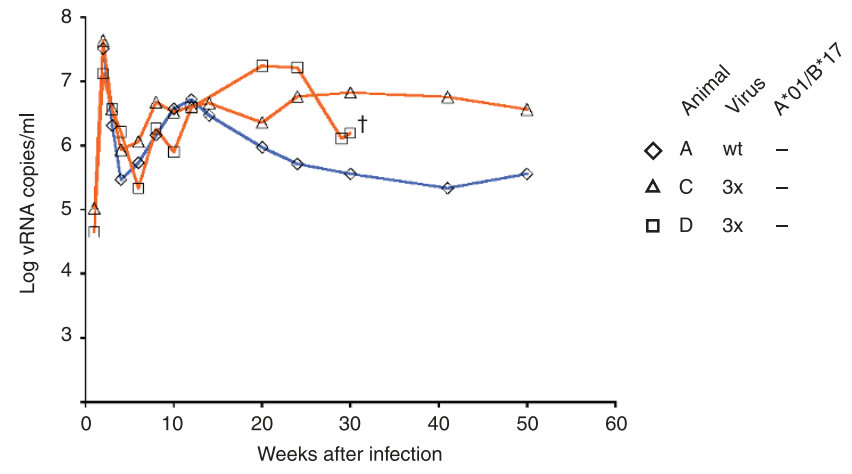

Figure $33 x$ SIV replication in vivo. (a) Three of four Mamu-A*01/B*17 double-positive macaques effectively controlled 3x SIV infection. (b) 3x SIV replicates to high titer in Mamu-A*01/B*17 double-negative animals. Virus concentrations are shown as viral RNA genomes (vRNA copies) per ml plasma. Blue traces, animals infected with wild-type SIV mac239; $_{\text {; }}$ orange traces, animals infected with $3 x$ SIV. Filled symbols represent double-positive animals; open symbols represent double-negative animals. Daggers indicate killing of animals D (30 weeks after infection) and E (40 weeks after infection).

We sequenced the complete genomes of viruses isolated from plasma at the time of necropsy in 35 animals (D.H.O., K. Krebs, E.J.D. and D.I.W., unpublished observations). In doing so, we did not detect mutations outside these epitopes occurring in association with intraepitopic variation, except for those mutations that were associated with escape in Gag CM9 as discussed above. We therefore conclude that it is unlikely that we could not detect other compensatory substitutions that might enhance the fitness of viral escape variants in vivo.

\section{Triple-mutant virus shows reduced fitness in vitro}

We next produced a clonal virus encoding escape mutations in all three epitopes ( $3 \mathrm{x}$ SIV) and characterized its in vitro growth properties. When the same dose of wild-type or $3 \mathrm{x}$ SIV was used to infect macaque peripheral blood mononuclear cells (PBMCs), $3 \mathrm{x}$ SIV replication lagged behind that of wild-type SIV in the first $96 \mathrm{~h}$. After $5 \mathrm{~d}$ in culture, however, the two viruses attained similar titers (Fig. 2a). These results suggest that $3 \mathrm{x}$ SIV replication is less efficient than that of the wild-type virus. To compare the fitness levels of the two viruses more directly, we devised a direct viral competition assay in which macaque PBMCs were infected with mixtures of the two virus stocks, totaling $10 \mathrm{ng}$ of p27. Replication of each virus species in the culture was detected using a quantitative RT-PCR technique with sequence-specific primers (SSP) that specifically targeted the wild-type nef epitope sequence. Because the quantitative RT-PCR/SSP reaction was inherently less efficient than the nonselective reaction, we could not effectively discriminate between wild-type and 3x SIV in the first $48 \mathrm{~h}$ of infection, when total virus concentrations were low. However, the direct com-

Figure 4 Epitope mutations are largely preserved in Mamu- $A * 01 / B^{*} 17$ double-positive macaques infected with $3 x$ SIV. (a-c) Sequences of Gag CM9 (a), Tat SL8 (b) and Nef IW9 (c) epitopes. MHC class I genotype of each animal (determined by PCR/SSP) is indicated. Data shown are result of direct sequencing of bulk viral cDNA amplicons. Lower-case letters indicate amino acid encoded by predominant codon at sites of mixed-base nucleotide heterogeneity. $X$ denotes site of mixed-base heterogeneity with no dominant codon. Nef YY9 (YTSGPGIRY) is underlined in each animal. Green boxes indicate amino acid variation consistent with escape from Mamu-A*02restricted, Nef YY9-specific CTLs, which we observed in a previous study ${ }^{32}$. petition assays showed that after $5 \mathrm{~d}$ in culture, wild-type SIV had completely outgrown the mutant when the inocula consisted of equal amounts of the two viruses (Fig. 2b). In contrast, 3x SIV remained predominant throughout the culture period when the inoculum ratios favored it, indicating that $3 \mathrm{x}$ SIV can outgrow wild-type virus, but only when inoculated in excess. We therefore concluded that growth of $3 \mathrm{x}$ SIV is impaired compared with wildtype $\mathrm{SIV}_{\text {mac239. }}$

\begin{tabular}{|c|c|c|c|c|}
\hline \multirow{4}{*}{$\begin{array}{l}\text { a } \\
\text { Gag CM9 } \\
\text { wild type } \\
\text { Inoculum }\end{array}$} & $E$ & $F$ & $\mathrm{G}$ & $\mathrm{H}$ \\
\hline & $A^{\star} 01 B^{\star} 17$ & $A^{*} 01 B^{*} 17$ & $A^{*} 01 A^{*} 02 B^{*} 17$ & $A^{\star} 01 B^{\star} 17$ \\
\hline & I CTPYDINQM I & I CTPYDINQM I & I CTPYDINQM I & I CTPYDINQM I \\
\hline & $\mathrm{v} \cdot \mathrm{A} \ldots \ldots, \mathrm{V}$ & $\mathrm{v} \cdot \mathrm{A} \ldots \ldots, \mathrm{V}$ & $\mathrm{v} . \mathrm{A} \ldots \ldots, \mathrm{V}$ & $\mathrm{V} \cdot \mathrm{A} \ldots \ldots, \mathrm{V}$ \\
\hline Week 2 & $\mathrm{v} . \mathrm{A} \ldots \ldots, \mathrm{V}$ & $\mathrm{V} \cdot \mathrm{A} \ldots \ldots, \mathrm{V}$ & $\mathrm{V} \cdot \mathrm{A} \ldots \ldots, \mathrm{V}$ & $\mathrm{V} . \mathrm{A} \ldots \ldots \mathrm{V}$ \\
\hline Week & $\mathrm{v} . \mathrm{A} \ldots \ldots \mathrm{V}$ & $\mathrm{V} \cdot \mathrm{A} \ldots \ldots, \mathrm{V}$ & $\mathrm{V} \cdot \mathrm{A} \ldots \ldots \mathrm{V}$ & $\mathrm{v} . \mathrm{A} \ldots \ldots \mathrm{V}$ \\
\hline Week & $\mathrm{v}, \mathrm{A} \ldots \ldots, \mathrm{V}$ & $\mathrm{V} \cdot \mathrm{A} \ldots \ldots, \mathrm{V}$ & $\mathrm{V} \cdot \mathrm{A} \ldots \ldots, \mathrm{V}$ & $\mathrm{v} \cdot \mathrm{A} \ldots \ldots, \mathrm{v}$ \\
\hline Week $1 \mathrm{C}$ & $\mathrm{V} \cdot \mathrm{A} \ldots \ldots, \mathrm{V}$ & $\mathrm{V} \cdot \mathrm{A} \ldots \ldots, \mathrm{V}$ & & $\mathrm{V} . \mathrm{A} \ldots \ldots, \mathrm{V}$ \\
\hline Week 1 & $\mathrm{v} . \mathrm{A} \ldots \ldots, \mathrm{v}$ & $\mathrm{v} \cdot \mathrm{A} \ldots \ldots, \mathrm{v}$ & $\mathrm{v} \cdot \mathrm{A} \ldots \ldots, \mathrm{V}$ & \\
\hline Week $2 \mathrm{C}$ & $\mathrm{v}, \mathrm{A}, \ldots \ldots \mathrm{V}$ & & $\mathrm{v} \cdot \mathrm{A}, \ldots \ldots \mathrm{V}$ & $\mathrm{v} \cdot \mathrm{A} \ldots \ldots, \mathrm{v}$ \\
\hline Week 2 & $\mathrm{v} . \mathrm{A} \ldots \ldots \mathrm{v}$ & $\mathrm{v} \cdot \mathrm{A} \ldots \ldots, \mathrm{v}$ & $\mathrm{v} \cdot \mathrm{A} \ldots \ldots \mathrm{v}$ & \\
\hline Week $3 C$ & $\mathrm{~V}, \mathrm{~A} \ldots \ldots, \mathrm{V}$ & & & \\
\hline Week 3 & & $\mathrm{~V} \cdot \mathrm{A} \ldots \ldots, \mathrm{V}$ & $\mathrm{V} \cdot \mathrm{A} \ldots \ldots, \mathrm{V}$ & \\
\hline Week 3 & $\mathrm{v}, \mathrm{A}, \ldots \ldots \mathrm{v}$ & & & \\
\hline \multirow{2}{*}{\multicolumn{5}{|c|}{$\mathbf{b}_{\text {Tat SL8 }}$}} \\
\hline & & & & \\
\hline wild type & STPESANL & STPESANL & STPESANL & STPESANL \\
\hline Inoculun & $P \ldots \ldots Q$ & P....Q & P.W.Q & P.W.Q \\
\hline Week & P....Q & P...QQ & & P.W.Q \\
\hline Week & P.W.Q & $P \ldots \ldots Q$ & P.W.Q & $P \ldots \cdots Q$ \\
\hline Week & P.W.Q & P...QQ & P...QQ & \\
\hline Week 1 & P....Q & P....Q & P....Q & P....Q \\
\hline Week 1 & Pt....Q & P....Q & & \\
\hline Week $2 C$ & P....Q & P....Q & P....Q & \\
\hline Week 2 & P. $\ldots . Q$ & P. $\cdots \cdot Q$ & P....Q & \\
\hline Week $3 c$ & P....Q & & & \\
\hline Week 3 & & P.W. Q & P..... & \\
\hline Week 35 & P.W.Q & & & \\
\hline \multicolumn{5}{|l|}{ C } \\
\hline Nef IWS & $A^{*} 02$ & $A^{*} 02$ & $A^{*} 02$ & $A^{*} 02$ \\
\hline wild type & IRYPKTFGW & IRYPKTFGW & IRYPKTFGW & IRYPKTFGW \\
\hline Inoculur & 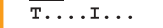 & 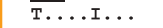 & $\overline{\text { T. } \ldots I \ldots ~}$ & 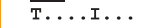 \\
\hline Week & T...I. . & T.... I.. & T.... I... & T...I. . \\
\hline Week & T...... & т....... & T...I... & T...I. \\
\hline Week & т...... & т....i... & T....... & $x \ldots x \ldots$ \\
\hline Week 1 & т....... & T...t... & $x \ldots x \ldots$ & т...... \\
\hline Week 1 & т....i... & т....... & т........ & \\
\hline Week $2 C$ & t....x... & $\mathrm{x} \ldots \ldots \ldots$ & P...... & T.X..X... \\
\hline Week 2 & т....... & т....... & P...... & \\
\hline Week $3 \mathrm{C}$ & т...M... & & & \\
\hline Week 3 & & $x \ldots \ldots$ & P.. & \\
\hline Week 3 & т....... & & & \\
\hline
\end{tabular}


Figure 5 Two of three $3 x$ SIV epitope sequences evolve toward wild type in Mamu- $A * 01 / B * 17$ double-negative macaques. (a-c) Sequences of Gag CM9 (a), Tat SL8 (b) and Nef IW9 (c) epitopes. Black boxes around animal names indicate Mamu-A*01/B*17 double-negative. Data are presented as in

Figure 4. Colored borders indicate strain of SIV used to challenge each animal (blue, wild-type SIV; orange, 3x SIV). Replacements consistent with escape from Mamu-A*02-restricted epitope that overlaps Nef IW9 are boxed in green.

\section{3x SIV mutations are preserved in MHC-similar animals}

To assess the in vivo replicative potential of $3 \mathrm{x}$ SIV and model the fate of epitope mutations in HLA-similar hosts, we infected four Mamu$A^{\star} 01 / B^{\star} 17$ double-positive macaques with $3 x$ SIV. As controls, we also infected one Mamu- $A^{\star} 01 / B^{\star} 17$ double-negative animal $(A)$ and one double-positive animal (B) with the same dose of wild-type $\mathrm{SIV}_{\text {mac239. }}$ Both viruses reached titers greater than $10^{6}$ viral RNA (vRNA) copies per ml plasma in every animal. Notably, three of four double-positive macaques maintained 3x SIV viral loads below 100,000 vRNA copies per $\mathrm{ml}$ in the chronic phase (Fig. 3a), in contrast to double-negative animals, whose chronic-phase virus loads were at least tenfold higher (Fig. 3b). However, CTL responses directed against the mutant epitopes were barely detectable in these animals throughout infection (data not shown). Furthermore, the engineered escape mutations in $3 \mathrm{x}$ SIV Gag and Tat were preserved throughout our study period in all double-positive animals (Fig. 4a,b). In contrast, the mutant Nef IW9 sequence continued to evolve in each of these animals (Fig. 4c). This result agrees with previously reported observations of maternal-tofetal transmission of an escape variant of HIV-1 (ref. 26). In that study, HLA-B27-positive infants did not recognize a variant HLA-B27restricted epitope present in the virus transmitted by their B27positive mothers, and the transmitted escape variant sequence persisted for several years.

\section{Reversion of 3x SIV epitopes in MHC-disparate animals}

Because HIV is most often transmitted between HLA class I-disparate individuals, we next inoculated two Mamu- $\mathrm{A}^{\star} 01 / \mathrm{B}^{\star} 17$ double-negative macaques (animals $\mathrm{C}$ and D) with $3 \mathrm{x}$ SIV. Peak $3 \mathrm{x}$ SIV viremia in these animals was greater than $10^{7}$ copies per ml plasma, similar to that observed in the Mamu- $\mathrm{A}^{\star} 01 / \mathrm{B}^{\star} 17$-double-negative animal (A) infected with wild-type $\operatorname{SIV}_{\text {mac239 }}$ (Fig. 3b). Animals A and C had clinical courses typical of macaques previously infected with SIV $_{\text {mac239 }}$ in our laboratory. In contrast, animal D's course was similar to that of rapid progressors, and showed severe thrombocytopenia and a CD4 ${ }^{+}$ cell count $<500 / \mu \mathrm{l}$ when killed 30 weeks after infection. However, although animal C survived much longer, $3 x$ SIV caused persistently high viremia $\left(>10^{6}\right.$ copies $\left./ \mathrm{ml}\right)$ during the chronic phase of infection in both double-negative macaques challenged with this stock.

$3 x$ SIV epitope sequences evolved rapidly in the double-negative animals. The wild-type Gag CM9 epitope sequence was completely reestablished in animals $\mathrm{C}$ and D by 16 weeks after infection (Fig. 5a). The sequence of the Nef IW9 epitope also evolved toward wild type during the first 16 weeks of infection in each double-negative macaque (Fig. 5c). Coincident escape from a Mamu-A*02-restricted CTL response to an overlapping epitope (Nef YY9; ref. 32) also resulted in new substitutions in this region (Fig. 5b). Thus, there seems to be a strong selective advantage favoring the wild-type sequences in both the Nef IW9 and Gag CM9 epitopes in vivo, reflecting the reduced fitness of $3 \mathrm{x}$ SIV we observed in vitro. Indeed, our attempts to produce viruses harboring the single mutation encoding T182A (CAPYDINQM) were unsuccessful, suggesting that without the compensatory substitutions flanking the epitope, this mutation exacts a catastrophic cost to viral replication. Recent experimental evidence from our labo-

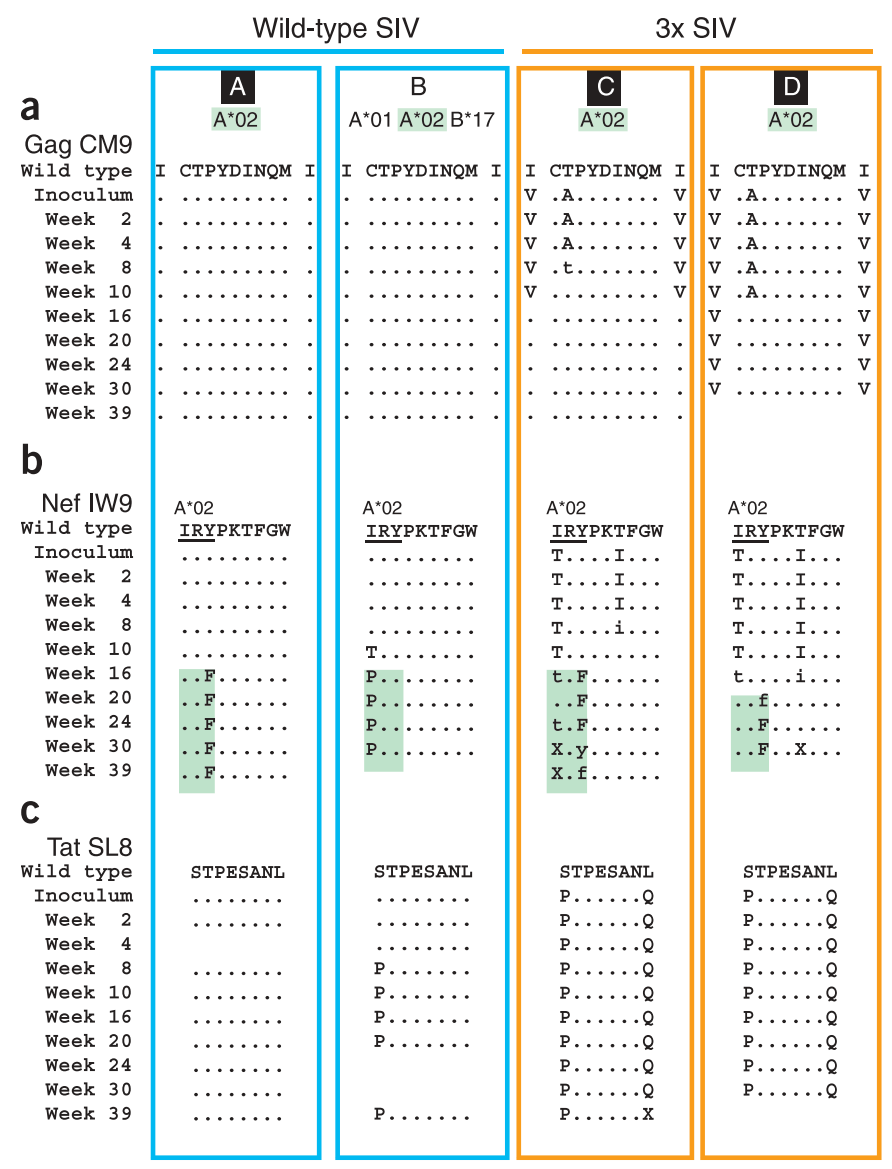

ratory ${ }^{30}$ and others ${ }^{31,33,34}$ supports this conclusion. In contrast, mutations introduced in the rapidly escaping Tat SL8 epitope were preserved in animal D throughout the study period (Fig. 5c), and a complex population of sequences was detected in this epitope in animal C beginning 30 weeks after infection. The composition of Tat SL8 sequences in animal $\mathrm{C}$ late in infection was reminiscent of that found in Mamu-A*01-negative animals infected with $\operatorname{SIV}_{\mathrm{ppm}}$ (Fig. 1c). Escape mutations in Tat SL8 may also reduce fitness in vivo, but are likely to be better tolerated than mutations affecting the Nef IW9 or Gag CM9 epitopes. Together, our results suggest that mutations in CTL epitopes can have moderate to severe negative effects on viral replicative fitness.

\section{DISCUSSION}

We have shown that SIV CTL escape variants can revert to wild-type sequences after infecting new hosts possessing disparate MHC class I alleles. We also observed an apparent cost to replicative fitness in a SIV $_{\text {mac239 }}$ variant engineered to contain CTL escape mutations. These findings led us to hypothesize that CTL pressure often selects for escape mutations in infected individuals, but some of these escape variant sequences will not be stably propagated as viruses circulate in HLA-diverse populations. In our study, the heterogeneous $\mathrm{SIV}_{\mathrm{ppm}}$ isolate harbored mutations in Mamu- $\mathrm{A}^{\star} 02$ - and $\mathrm{B}^{\star} 17$-restricted CTL epitopes that evolved toward wild-type $\operatorname{SIV}_{\text {mac239 }}$ sequences in macaques that did not express these molecules. To rigorously test the hypothesis that CTL escape mutations can reduce viral fitness, we engineered a clonal virus encoding three CTL escape variants. Replication of this cloned escape mutant virus, 3x SIV, was reduced in vitro with respect to the wild-type virus, indicating that incorporation 
of one or more epitope mutations into the $\mathrm{SIV}_{\text {mac239 }}$ sequence reduced viral fitness. Indeed, the mutation in Gag CM9 in particular seems to have a profound negative effect on viral fitness ${ }^{30,31,33,34}$. Accordingly, wild-type Gag CM9 and Nef IW9 epitope sequences were reestablished in Mamu- $A^{\star} 01$ - and $B^{\star} 17$-negative animals within 16 weeks of infection with the molecularly cloned $3 x$ SIV. In contrast, the mutant Tat SL8 epitope sequence of 3x SIV did not revert to wild type in the absence of immune selection, but instead showed some sequence oscillation, suggesting that variation is better tolerated in this epitope. Escape mutations in Tat SL8 underwent similar oscillations in Mamu- $A^{\star} 01$-negative animals infected with $\operatorname{SIV}_{\mathrm{ppm}}$, in agreement with this hypothesis.

It is likely that escape variants will only outgrow the wild-type virus when the benefit of escape from a particular CTL response outweighs the loss of fitness. However, it is likely that multiple interacting determinants affect the selection of CTL escape viruses. We have previously observed that rapidly escaping CTL epitopes tend to be the targets of particularly high-avidity CTLs ${ }^{23}$. These epitopes also cluster in regulatory and accessory proteins. Variation in these sequences may therefore incur lower fitness costs than variations in epitopes derived from structurally or functionally conserved regions. Furthermore, escape from CTLs that target particularly conserved regions may involve several substitutions, including extraepitopic substitutions that compensate for the loss of fitness resulting from escape. The outgrowth of CTL escape variants therefore probably represents the additive effects of several virological and immunological parameters, including CTL frequency and efficacy, the number of nucleotide substitutions required for effective escape, and the costs of these substitutions to fitness and viral replication. We expect this constellation of factors to be different for each CTL epitope; their differential effects will determine whether escape mutations will be lost or preserved upon transmission to new hosts.

It is heartening to believe that epitopes targeted by effective CTLs may not be entirely lost from circulating virus sequences as the HIV epidemic progresses. Successful vaccines for HIV will probably need to stimulate CTL responses. Unfortunately, the propensity of CTLs to select for escape variant viruses, and the possibility that these variants can be propagated in human populations, represent enormous potential obstacles to the efficacy of CTL-based vaccines. Our results show that escape mutations can revert to CTL-susceptible sequences when escape variant viruses are transmitted among MHC-disparate individuals, but we must be prudent in extending these results by analogy to humans infected with HIV. Set-point viremia with $\mathrm{SIV}_{\text {mac239 }}$ in Indian rhesus macaques is typically tenfold higher than that seen in HIVinfected humans, which may affect the rate of viral evolution in vivo. Moreover, macaques expressing Mamu- $A^{\star} 01$, especially in association with Mamu- $B^{\star} 17$, seem to be predisposed to unusually effective control of $\mathrm{SIV}_{\text {mac239 }}$ infection ${ }^{28}$. As a result, analogous studies in humans will be required to determine the extent to which HIV CTL epitope sequences will be preserved in circulating viruses.

Our results may also proffer criteria by which the efficacy of particular CTL responses can be assessed. Transmitted CTL escape mutations may evolve at different rates in the absence of immune selection in new hosts, as the regions from which they are derived are under different constraints. CTL-based vaccines may thus be most effective if they include epitopes in which escape mutations revert rapidly after transmission into new hosts. This strategy offers at least two potential benefits over current approaches. First, rapid re-establishment of CTL-susceptible sequences suggests that targeted epitopes will persist in circulating virus sequences. Second, such rapid evolution also implies a particularly large fitness cost associated with those escape mutations. Viral escape from CTL targeting of such regions may therefore involve particularly drastic reductions in fitness, 'crippling' the replicating virus population and facilitating control of infection. Vaccine-induced CTL responses that drive the evolution of viral variants with low replicative fitness could offer hope for immune control and amelioration of HIV disease.

\section{METHODS}

Animals. Indian rhesus macaques (Macaca mulatta) from the Wisconsin National Primate Research Center colony were typed for MHC class I alleles Mamu- $A^{\star} 01, A^{\star} 02, A^{\star} 08, A^{\star} 11, B^{\star} 01, B^{\star} 03, B^{\star} 04, B^{\star} 17$ and $B^{\star} 29$ by sequencespecific $\mathrm{PCR}^{35}$. Animals were cared for according to regulations of the Guide for the Care and Use of Laboratory Animals of the National Research Council, as approved by the University of Wisconsin Institutional Animal Care and Use Committee.

Viruses and infections. Animals were infected with the heterogeneous isolate $\mathrm{SIV}_{\mathrm{ppm}}$ in a previous study ${ }^{19}$. The passage history of SIV $_{\mathrm{ppm}}$ has not been well characterized, but sequencing and phylogenetic analyses suggest that it is the result of a low number of passages of cloned $\operatorname{SIV}_{\text {mac239 }}$ through macaques expressing common MHC class I molecules (Supplementary Fig. 1 online). Wild-type and $3 \mathrm{x} \mathrm{SIV}_{\text {mac239 }}$ were produced by transfection of Vero cells with plasmid DNA encoding proviral sequences. CEMx174 cells were added to the Vero cultures $1 \mathrm{~d}$ after transfection and removed to flasks $3 \mathrm{~d}$ after transfection. Viruses were then amplified on CEMx174 cells, and cell-free supernatant was collected $2 \mathrm{~d}$ after peak syncytium formation. Direct sequencing of RT-PCR amplicons representing all $3 \mathrm{x}$ SIV open reading frames (as described previously ${ }^{23}$ ) confirmed that this stock harbored only the engineered nucleotide substitutions and was otherwise identical to $\operatorname{SIV}_{\text {mac239 }}$ (data not shown). Animals were challenged intravenously with $100 \mathrm{TCID}_{50}$ of either virus stock. Plasma virus load was determined by the branched-chain DNA assay (Bayer Diagnostics).

Viral competition assay. Two million phytohemagglutinin-stimulated rhesus macaque PBMCs were infected in triplicate, as described above, with $10 \mathrm{ng}$ p27 total virus containing wild-type $\mathrm{SIV}_{\text {mac239 }}$ or $3 \mathrm{x}$ SIV alone or together. Cultures were maintained for $5 \mathrm{~d}$, and supernatants were sampled every $24 \mathrm{~h}$ for the duration of the assay. Results shown in Figure $2 \mathrm{~b}$ are representative of two separate assays using two independently prepared stocks of $3 \mathrm{x}$ SIV. We determined the proportion of wild-type vRNA in each sample using a modification of a published method $^{36}$. Total vRNA in supernatants was measured using a nonselective quantitative PCR assay with primers 9700-F (5'-TAAAAGAAAAGGGGGGACTG-3') and nef-R (5'-CCTCATCCTCCTGTGC- $3^{\prime}$ ). For selective amplification of wild-type $\operatorname{SIV}_{\text {mac239 }}$ vRNA in the presence of $3 x$ SIV vRNA, we used primer 9700-F with a sequence-specific primer, SIV 9845-R (5'AAATGTCTTTGGGTATCTAA-3'), targeting the two-nucleotide polymorphisms in the nef coding region. Reverse transcription and amplification were done in a single reaction using the Roche SYBR Green RNA Master Probes kit. Reaction conditions were optimized for primer concentration, MnOAc concentration and annealing temperature. The sequence-specific primer $9845-\mathrm{R}$ would not anneal to $3 \mathrm{x}$ SIV vRNA above $59^{\circ} \mathrm{C}$ at any concentration of primer or MnOAc tested. Reactions were done in the Roche Lightcycler, using the following cycling parameters: $61^{\circ} \mathrm{C}$ for $25 \mathrm{~min}$ (at which temperature wild-type vRNA is selectively reverse transcribed with primer $9845-\mathrm{R}), 95^{\circ} \mathrm{C}$ for $30 \mathrm{~s}$, and 45 cycles of $95{ }^{\circ} \mathrm{C}$ for $1 \mathrm{~s}, 58^{\circ} \mathrm{C}$ for $10 \mathrm{~s}, 72^{\circ} \mathrm{C}$ for $10 \mathrm{~s}$ and $77^{\circ} \mathrm{C}$ for $2 \mathrm{~s}$. Fluorescence was measured at $71^{\circ} \mathrm{C}$ at the end of every PCR cycle. Melt curves were obtained for every sample after PCR. The number of cycles required to reach threshold fluorescence was determined, and the number of vRNA copies initially present was calculated by extrapolation onto a standard curve prepared from synthetic dilutions of wild-type vRNA. All reactions were done in triplicate, with the mean of the values used for calculations. Our threshold of detection with this method was $0.08 \%$ wild-type vRNA in 2 million copies of $3 \mathrm{x}$ SIV vRNA (data not shown).

Viral sequence analysis. Amplification of viral cDNA by RT-PCR was done as described previously ${ }^{23}$, using viral genomic RNA isolated from animal plasma or tissue culture supernatants. We used three previously described primer 
pairs $^{23}$ to amplify regions surrounding the sequences encoding Gag CM9, Tat SL8 and Nef IW9. PCR products were directly sequenced on an ABI 377 or ABI 3730 automated sequencer. Mixed bases and nucleotide replacements were detected in sequence data using Sequencher and MacVector 7.0 software.

Note: Supplementary information is available on the Nature Medicine website.

\section{ACKNOWLEDGMENTS}

We gratefully acknowledge J. Helgeland, J. Mitchen, C. Bunger, K. Vielhuber and E. Rakasz; I. Bolton and the veterinarians and technicians of the WNPRC; and T. Jacoby and W. Rehrauer for their work in support of this study. We also thank W. Rehrauer for critical review of this manuscript. This work was supported by National Institutes of Health grants RO1-AI-46366, RO1-AI-49120 and RO1-AI52056 to D.I.W., and P51 RR001676-43 to the WNPRC. D.I.W. is an Elizabeth Glaser Scientist. A.S. is supported by NIH-NIAID contract NO1-AI-95362.

\section{COMPETING INTERESTS STATEMENT}

The authors declare that they have no competing financial interests.

Received 30 October 2003; accepted 26 January 2004

Published online at http://www.nature.com/naturemedicine/

1. Burton, D.R. Antibodies, viruses and vaccines. Nat. Rev. Immunol. 2, 706-713 (2002).

2. Ho, D.D. \& Huang, Y. The HIV-1 vaccine race. Cell 110, 135-138 (2002).

3. Richman, D.D., Wrin, T., Little, S.J. \& Petropoulos, C.J. Rapid evolution of the neutralizing antibody response to HIV type 1 infection. Proc. Natl. Acad. Sci. USA 100, 4144-4149 (2003).

4. Wei, X. et al. Antibody neutralization and escape by HIV-1. Nature 422, 307-312 (2003).

5. McMichael, A.J. \& Rowland-Jones, S.L. Cellular immune responses to HIV. Nature 410, 980-987 (2001)

6. Walker, B.D. \& Korber, B.T. Immune control of HIV: the obstacles of HLA and vira diversity. Nat. Immunol. 2, 473-475 (2001).

7. McMichael, A.J. \& Hanke, T. HIV vaccines 1983-2003. Nat. Med. 9, 874-880 (2003).

8. Borrow, P., Lewicki, H., Hahn, B.H., Shaw, G.M. \& Oldstone, M.B. Virus-specific $\mathrm{CD}^{+}$cytotoxic T-lymphocyte activity associated with control of viremia in primary human immunodeficiency virus type 1 infection. J. Virol. 68, 6103-6110 (1994).

9. Koup, R.A. et al. Temporal association of cellular immune responses with the initial control of viremia in primary human immunodeficiency virus type 1 syndrome. J. Virol. 68, 4650-4655 (1994).

10. Jin, $X$. et al. Dramatic rise in plasma viremia after $C D 8^{+} \mathrm{T}$ cell depletion in simian immunodeficiency virus-infected macaques. J. Exp. Med. 189, 991-998 (1999).

11. Matano, T. et al. Administration of an anti-CD8 monoclonal antibody interferes with the clearance of chimeric simian/human immunodeficiency virus during primary infections of rhesus macaques. J. Virol. 72, 164-169 (1998).

12. Schmitz, J.E. et al. Control of viremia in simian immunodeficiency virus infection by CD8+ lymphocytes. Science 283, 857-860 (1999).

13. Phillips, R.E. et al. Human immunodeficiency virus genetic variation that can escape cytotoxic T cell recognition. Nature 354, 453-459 (1991).

14. Koenig, S. et al. Transfer of HIV-1-specific cytotoxic T lymphocytes to an AIDS patient leads to selection for mutant HIV variants and subsequent disease progression. Nat. Med. 1, 330-336 (1995).

15. Wolinsky, S.M. et al. Adaptive evolution of human immunodeficiency virus-type 1 during the natural course of infection. Science 272, 537-542 (1996)

16. Borrow, P. et al. Antiviral pressure exerted by HIV-1-specific cytotoxic T Iymphocytes
(CTLs) during primary infection demonstrated by rapid selection of CTL escape virus. Nat. Med. 3, 205-211 (1997).

17. Goulder, P.J. et al. Late escape from an immunodominant cytotoxic T-lymphocyte response associated with progression to AIDS. Nat. Med. 3, 212-217 (1997).

18. Price, D.A. et al. Positive selection of HIV-1 cytotoxic T lymphocyte escape variants during primary infection. Proc. Natl. Acad. Sci. USA 94, 1890-1895 (1997).

19. Evans, D.T. et al. Virus-specific cytotoxic T-lymphocyte responses select for aminoacid variation in simian immunodeficiency virus env and Nef. Nat. Med. 5, 1270-1276 (1999).

20. Allen, T.M. et al. Tat-specific cytotoxic T Iymphocytes select for SIV escape variants during resolution of primary viraemia. Nature 407, 386-390 (2000).

21. Kelleher, A.D. et al. Clustered mutations in HIV-1 gag are consistently required for escape from HLA-B27-restricted cytotoxic T lymphocyte responses. J. Exp. Med. 193, 375-386 (2001).

22. McMichael, A. T cell responses and viral escape. Cell 93, 673-676 (1998)

23. O'Connor, D.H. et al. Acute phase cytotoxic T lymphocyte escape is a hallmark of simian immunodeficiency virus infection. Nat. Med. 8, 493-499 (2002).

24. Moore, C.B. et al. Evidence of HIV-1 adaptation to HLA-restricted immune responses at a population level. Science 296, 1439-1443 (2002).

25. Barouch, D.H. et al. Eventual AIDS vaccine failure in a rhesus monkey by viral escape from cytotoxic T lymphocytes. Nature 415, 335-339 (2002).

26. Goulder, P.J. et al. Evolution and transmission of stable CTL escape mutations in HIV infection. Nature 412, 334-338 (2001).

27. Regier, D.A. \& Desrosiers, R.C. The complete nucleotide sequence of a pathogenic molecular clone of simian immunodeficiency virus. AIDS Res. Hum. Retroviruses 6 , 1221-1231 (1990).

28. O'Connor, D.H. et al. Major histocompatibility complex class I alleles associated with slow simian immunodeficiency virus disease progression bind epitopes recognized by dominant acute-phase cytotoxic-T-lymphocyte responses. J. Virol. 77, 9029-9040 (2003)

29. Mothe, B.R. et al. Dominance of CD8 responses specific for epitopes bound by a single major histocompatibility complex class I molecule during the acute phase of viral infection. J. Virol. 76, 875-884 (2002).

30. Friedrich, T.C. et al. Extra-epitopic compensatory substitutions restore fitness to simian immunodeficiency virus variants that escape from an immunodominant cytotoxic T-lymphocyte response. J. Virol. (in the press).

31. Peyerl, F.W. et al. Simian-human immunodeficiency virus escape from cytotoxic Tlymphocyte recognition at a structurally constrained epitope. J. Virol. 77, $12572-12578$ (2003).

32. Vogel, T.U. et al. Escape in one of two cytotoxic T-lymphocyte epitopes bound by a high-frequency major histocompatibility complex class I molecule, Mamu-A*02: a paradigm for virus evolution and persistence? J. Virol. 76, 11623-11636 (2002).

33. Rue, S.M., Roos, J.W., Amzel, L.M., Clements, J.E. \& Barber, S.A. Hydrogen bonding at a conserved threonine in lentivirus capsid is required for virus replication. J. Virol. 77, 8009-8018 (2003).

34. von Schwedler, U.K., Stray, K.M., Garrus, J.E. \& Sundquist, W.I. Functional surfaces of the human immunodeficiency virus type 1 capsid protein. J. Virol. 77 5439-5450 (2003).

35. Knapp, L.A., Lehmann, E., Hennes, L., Eberle, M.E. \& Watkins, D.I. High-resolution HLA-DRB typing using denaturing gradient gel electrophoresis and direct sequencing. Tissue Antigens 50, 170-177 (1997).

36. Hance, A.J. et al. Changes in human immunodeficiency virus type 1 populations after treatment interruption in patients failing antiretroviral therapy. J. Virol. 75, 6410-6417 (2001)

37. Sidney, J. et al. Definition of the Mamu $A^{*} 01$ peptide binding specificity: application to the identification of wild-type and optimized ligands from simian immunodeficiency virus regulatory proteins. J. Immunol. 165, 6387-6399 (2000).

38. Mothe, B.R. et al. Characterization of the peptide-binding specificity of Mamu- ${ }^{*} 17$ and identification of Mamu-B*17-restricted epitopes derived from simian immunodeficiency virus proteins. J. Immunol. 169, 210-219 (2002). 